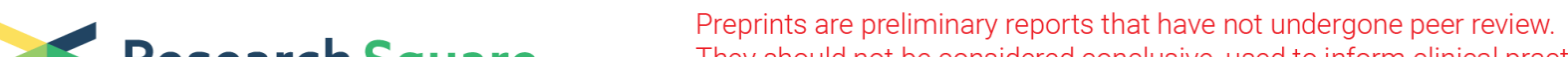 $\begin{array}{ll}\text { Research Square } & \text { They should not be considered conclusive, used to inform clinical practice, } \\ \text { or referenced by the media as validated information. }\end{array}$
}

\section{Evaluating the national implementation of Bright IDEAS in clinical settings: applying the RE-AIM framework to characterize the clinical perspective}

Demetria McNeal ( $\nabla$ demetria.mcneal@cuanschutz.edu)

University of Colorado Denver - Anschutz Medical Campus https://orcid.org/0000-0002-7415-8631

O.J. Sahler

University of Rochester School of Medicine and Dentistry

Robert B. Noll

University of Pittsburgh

Diane L. Fairclough

University of Colorado Denver Health Sciences Center: University of Colorado - Anschutz Medical Campus

Megan E. Voll

University of Pittsburgh

Shubha Bhat

University of Colorado Denver School of Pharmacy: University of Colorado Denver Skaggs School of

Pharmacy and Pharmaceutical Sciences

Elaine H. Morrato

Loyola University Chicago

\section{Research}

Keywords: Dissemination, cancer survivorship, psychosocial intervention, RE-AIM

Posted Date: May 7th, 2021

DOI: https://doi.org/10.21203/rs.3.rs-473855/v1

License: (1) This work is licensed under a Creative Commons Attribution 4.0 International License. Read Full License 


\section{Abstract}

Background Bright IDEAS: Problem-Solving Skills Training (Bright IDEAS), a psychosocial intervention, has shown to improve problem-solving skills and reduce emotional distress in caregivers of children recently diagnosed with cancer. Though efforts have been made to scale up cancer control interventions, there are limited data assessing the adoption of Evidence-Based Cancer Control Programs (EBCCP) into clinical practice. This study describes the barriers and facilitators to implementation experienced by practitioners trained to use Bright IDEAS in their clinical settings.

Methods A total of 209 Pediatric psychosocial oncology practitioners were trained through 10 workshops. Adaptations were made to the training agenda and curriculum based on practitioner feedback following implementation in local institutions. Practitioners were interviewed to understand their training experience and gain their perspective on the effectiveness of delivering Bright IDEAS in the clinical setting. The RE-AIM framework was used to guide the evaluation process we employed to assess the effectiveness of this national effort to disseminate Bright IDEAS.

Results Interviews were conducted with 47 practitioners. Practitioners in the pre-adaptation group reported the identification of a particular profile of a client as a facilitator to intervention use. Additionally, perceived rigidity of the protocol, lack of consistency in intervention use with clients, feelings of being overwhelmed by the client as rationale for lack of interest, and lack of multiple trained practitioners at institutions were identified as barriers to intervention use. Practitioners in the post-adaptation group reported similar identification of a profile for an appropriate client for the intervention, more usage of Bright IDEAS materials, implementation across multiple clinical settings, and expressed continued commitment to intervention use as well as discussion of internal training for colleagues.

Conclusions This study provides insight into how psychosocial practitioners adopt, implement, and maintain Bright IDEAS in the clinical setting and identified important considerations and needs for both practitioners and larger health care systems. It also provides some cautions to those who wish to promulgate evidencebased interventions.

\section{Contributions To The Literature}

- We disseminated a psychosocial intervention nationally. This paper describes the evaluation of local implementation and results from practitioner interviews expressing best practices and necessary program adaptations for success.

- This study helps address a gap in the literature by identifying key learnings from evaluating the application of a psychosocial intervention into routine clinical care.

- Our findings call for increased evaluation of intervention implementation of psychosocial interventions and highlights the need to identify program adaptations early and often to ensure success in real-world application.

\section{Background}


There is increasing urgency to address the gap between the generation of new knowledge and empirical evidence focusing on its application to routine clinical care. ${ }^{1,2}$ This urgency is fueled, in part, by marginal awareness and variable adoption of effective interventions, commonly referred to as evidence-based practices (EBP) that research produces. Oftentimes, EBPs are tested under conditions that are different from the clinical practice settings for which they are designed. As a result, clinician-preferred interventions, many of which either lack empirical evidence or are delivered in a substandard manner, are preferred to EBPs. ${ }^{2,3}$

In response to the limited uptake of EBPs in the clinical setting, the National Cancer Institute ( $\mathrm{NCI}) \mathrm{created}$ a searchable database of evidence-based cancer control programs known as Evidence-Based Cancer Control Programs (EBCCP; formerly, Research-Tested Intervention Programs (RTIPs)) (Evidence-Based Cancer Control Programs (EBCCP) website. National Cancer Institute. https://ebccp.cancercontrol.cancer.gov. Last Updated: 02/12/2020. Accessed on: 04/07/2021). The EBCCP is designed to provide clinical program planners and public health practitioners with easy and immediate access to program materials. Despite efforts to scale up cancer control interventions, there are limited data assessing the adoption of survivorship/supportive care EBCCPs into clinical practice despite public availability through the database. ${ }^{4}$

This study examines the national implementation of a EBCCPs designated psychosocial intervention, Bright IDEAS: Problem-Solving Skills Training (Bright IDEAS), shown to improve problem-solving skills and reduce emotional distress in caregivers of children recently diagnosed with cancer. ${ }^{5-8}$ Bright IDEAS received EBCC designation in 2013 and $\mathrm{NCl}$ tracking statistics indicate the Bright IDEAS webpage received approximately 470 views annually (averaging 2.7 minutes per page); only $40 \mathrm{CDs}$ of the intervention materials were requested over a 7-year period. These findings suggest that, despite $\mathrm{NCl}$ endorsement and the availability of Bright IDEAS through the EBCCs website for over 10 years and considerable evidence supporting its efficacy, the leap from research protocol to standard clinical care was likely not occurring.

To increase awareness of Bright IDEAS and facilitate adoption, an educational grant (R25CA65520) was obtained to train 200 pediatric psychosocial oncology practitioners from across the country and coach them in the use of the intervention. The main objective of the study presented here was to obtain information from practitioners trained to use Bright IDEAS; about the barriers and facilitators to implementation in their clinical settings. The specific research questions for this study were:

1. Does training psychosocial practitioners to become competent in delivery facilitate implementation of Bright IDEAS into clinical practice?

2. What factors affect the broader adoption, implementation, and maintenance of Bright IDEAS as an evidence-based intervention for widespread use in the clinical setting?

This study presents longitudinal follow-up findings from qualitative interviews conducted among practitioners who participated in the Bright IDEAS training program, all of whom had committed to deliver the intervention at their respective institutions after receiving training to use Bright IDEAS. RE-AIM constructs were used to explore implementation barriers and facilitators. ${ }^{9}$ The goal was to identify factors for improving the pragmatic application in real-world clinical settings to ensure future successful implementation.

\section{Methods}




\section{Bright IDEAS Intervention}

Bright IDEAS is a cognitive-behavioral coping skills training approach that allows caregivers to identify problems or challenges they are encountering that they wish to resolve (Fig. 1). No constraints are placed on the type of problem or challenge and, interestingly, the majority of selected problems are not related to pediatric cancer. ${ }^{5-7}$ Optimum engagement is gained by focusing on problems the caregiver identifies as particularly relevant to him or her and the family. This approach also enables the caregiver and interventionist to review the effectiveness of selected solutions over time and changing circumstances. Notably, improved problem-solving skills mediates less distress. ${ }^{6,8}$

Bright IDEAS has established clinical effectiveness among mothers of children with recently diagnosed cancer and has demonstrated dramatic and sustained improvement in caregiver mental well-being by decreasing negative affectivity as a result of improved problem-solving skills. ${ }^{5,8}$ Clinical trials have shown that Bright IDEAS is acceptable to caregivers and, when used in six to eight 30-to 60-minute face-to-face sessions, enhances problem-solving skills and alleviates distress in mothers of children recently diagnosed with cancer. ${ }^{5}$ In particular, Bright IDEAS has proven to be significantly more effective over time than the most common form of psychosocial support, non-directive supportive counseling. ${ }^{10}$

\section{Training Format}

Grant R25CA65520 was designed to train 200 pediatric psychosocial oncology professionals through 10 interactive workshops (2015-2018). Practitioner recruitment for the workshops was leveraged through networks of professional organizations intimately involved in pediatric oncology: Children's Oncology Group (COG), Association of Pediatric Oncology Social Workers (APOSW), the Association of Pediatric Hematology/Oncology Nurses (APHON), and the Society of Pediatric Psychology (SPP). The national reach of these groups creates the optimal environment to disseminate Bright IDEAS across the country and reach the approximately 200 pediatric oncology centers in North America.

The 11/2-day training workshops were held in conjunction with association national meetings and endorsed through co-advertising. The workshops included summary information about the three large multi-site randomized controlled trials conducted to date demonstrating the efficacy of Bright IDEAS; role plays to observe and practice administration of the intervention; and in-depth discussions about implementation at an attendee's specific home institution. Workshop participants received up to $\$ 1,000$ to reimburse expenses associated with travel and lodging.

The original training agenda was modeled from the research training protocol and is detailed in Appendix A. After completing training, practitioners were asked about their perspective on their ability to adopt, implement, and maintain Bright IDEAS at their institution. The aim of the initial qualitative interviews was to identify factors for improving training delivery to improve implementation of Bright IDEAS in real-world clinical settings. After feedback from practitioners following initial implementation, adaptations were made to the training agenda which are presented in Appendix B. Table 1 summarizes the modifications and their rationale. 
Table 1

Bright IDEAS training modifications.

\begin{tabular}{|c|c|c|}
\hline $\begin{array}{l}\text { Initial Training } \\
\text { Concerns }\end{array}$ & Adaptive Training Modifications & Rationale for training modifications \\
\hline \multirow{2}{*}{$\begin{array}{l}\text { Research intensive } \\
\text { pre-workshop } \\
\text { study materials }\end{array}$} & $\begin{array}{l}\text { Watch online training videos and } \\
\text { practice working through a challenge }\end{array}$ & $\begin{array}{l}\text { Streamline training process and reduce } \\
\text { learning materials }\end{array}$ \\
\hline & $\begin{array}{l}\text { Creation of "Bright IDEAS Essential } \\
\text { Elements" handout }\end{array}$ & $\begin{array}{l}\text { Clarify the transition from research to } \\
\text { clinical applicability }\end{array}$ \\
\hline \multicolumn{3}{|l|}{$\begin{array}{l}\text { Research-focused } \\
\text { implementation of } \\
\text { Bright IDEAS }\end{array}$} \\
\hline $\begin{array}{l}\text { Incorporating } \\
\text { Bright IDEAS into } \\
\text { clinical workflow }\end{array}$ & $\begin{array}{l}\text { Inclusion of clinical workflow role } \\
\text { plays }\end{array}$ & Improve clinical implementation \\
\hline Personal case & Patient/family-focused case studies & Increase clinical relevance \\
\hline $\begin{array}{l}\text { Lack of } \\
\text { institutional } \\
\text { support and post- } \\
\text { training }\end{array}$ & $\begin{array}{l}\text { Letter of supervisor support required } \\
\text { for attendance (participant } \\
\text { expectations outlined in letter) }\end{array}$ & $\begin{array}{l}\text { Emphasize managerial support of } \\
\text { training and follow-up consultation calls } \\
\text { to implement Bright IDEAS }\end{array}$ \\
\hline $\begin{array}{l}\text { clinician } \\
\text { participation }\end{array}$ & & \\
\hline
\end{tabular}

The first adaptation made to the Bright IDEAS training was to the role-playing sessions. Training now uses real-world, family-centered scenarios, as compared to practitioner-focused scenarios to demonstrate the use of Bright IDEAS. The scenarios now reflect the key characteristics and attributes of Bright IDEAS recipients as identified by practitioners. Second, there is the additional role play of situations faced by practitioners' patients. Third, it was reported that a heavy emphasis on the empirical evidence for the efficacy of Bright IDEAS may actually have been too intimidating and seemed to discourage practitioners from asking about modifications to the protocol. Therefore, Essential Elements of Bright IDEAS (see supplementary material) was developed to clarify the transition from research to clinical applicability and help stream-line materials used during sessions. Finally, practitioners expressed a strong need to have institutional and community support to sustain Bright IDEAS as an embedded part of usual practice. Accordingly, acceptance into Bright IDEAS training sessions now requires a formal written commitment from a supervisor that supports the trainee's ability to attend the training workshop, implement Bright IDEAS in their clinical setting, and participate in the four consultation conference calls after training. After completion of the adapted training schedule, practitioners were asked about their perspective on their ability to adopt, implement, and maintain Bright IDEAS in the clinical setting.

To facilitate implementation and promote trial, one-hour consultation conference calls were also held at 1, 2, 3, and 5 months after the workshop. Each call was attended by 3-5 workshop participants and led by 2 workshop leaders. All attendees were expected to participate and were asked to complete the intervention with at least three patients, caregivers, or patient-caregiver dyads over the 5-month course of the conference calls. The practitioner's experiences were discussed on the calls in a supportive peer mentorship atmosphere. 


\section{Study Population}

\section{National Training Workshops}

We purposively sampled psychosocial practitioners who completed the Bright IDEAS training and delivered the intervention. A total of 209 practitioners representing 47 unique institutions completed the training. The interviews were scheduled to provide data from 3,6 , and 12 months following training in two waves to reflect the effect of modifying the workshop presentations in response to attendee suggestions. The project was approved by the Colorado Combined Institutional Review Board (COMIRB).

\section{Practitioner Interviews}

Study participant recruitment occurred between January 2017 and March 2020: Pre-adaptation: Wave 1- more than 12 months since training $(N=11)$, Wave 2- between 6 and 12 months $(N=9)$, and Wave 3- less than 6 months $(\mathrm{N}=10)$. Post-adaptation: Wave 1- more than 12 months since training $(\mathrm{N}=6)$, Wave 2- between 6 and 12 months $(N=4)$, and Wave 3 - less than 6 months $(N=4)$. Practitioners were contacted via email and invited to participate in a 30-minute telephone interview. A random sampling of participants was contacted initially. Depending on response rate, the decision was made to continue or discontinue contact to secure additional interviews as needed. In total, 106 professionals were contacted, 68 responded to the study invitation (64\% response), and 44 were scheduled (65\% participation) for interviewing allowing up to three contact attempts. Non-participation by three providers who originally agreed to participate was due to scheduling challenges. An honorarium of a $\$ 25$ gift card was offered for participation.

\section{Semi-structured interviews}

Semi-structured interviews were conducted over the telephone in their office or other private setting by the first author (DMM) who had no prior relationship with any of the respondents (range: 24-47 minutes per interview). All interviews were audio-recorded and transcribed verbatim.

\section{Conceptual Framework For Evaluation}

The RE-AIM framework, which is recognized by the $\mathrm{NCl}$ as a leading implementation framework in cancer control research, was used to guide the evaluation process we employed to assess the effectiveness of this national effort to disseminate Bright IDEAS. ${ }^{9}$

The interview guide was developed to be consistent with dimensions of the RE-AIM framework and explored adoption, implementation, and maintenance of Bright IDEAS in the clinical setting. Table 3 gives example questions from the interview guide used to focus the conversation. The interview guide was used flexibly with respect to the order of questions and the depth to which some issues were explored. The guide was pilot tested with a small sample of psychosocial providers $(n=5)$ prior to finalization. 
Table 3

Examples of interview questions.

\section{RE-AIM Example of questions dimensions}

Adoption

Would you consider Bright IDEAS to be a completely new approach to working with parents with a child newly diagnosed with cancer?

Probe: If yes, ask: how so? If no, ask: what is it most similar to that you have used or are more familiar with?

Implementation Please tell me about the ways you deliver Bright IDEAS to your clients?

Probe: How do you select appropriate clients for Bright IDEAS?

Probe: How do you determine the number of sessions? How do you determine how long the sessions should be?

Maintenance

What factors might influence your (dis) continuation of Bright IDEAS?

Probe: How significant a role does [insert factors stated by practictionr] play in determining your future use of Bright IDEAS?

\section{Data Analyses}

Analyses were completed using data analysis package ATLAS.ti 8.0 (Scientific Software Development GmbH, Berlin, Germany) for coding by study authors (DMM, SB) who are PhD and PharmD trained researchers with experience in qualitative methods, health services research, and D\&I science. All of the transcripts were double coded. The coders familiarized themselves with the data by carefully reading the transcripts. They then deductively coded the data using the constructs of the three RE-AIM dimensions: adoption, implementation, and maintenance. Discrepancies were resolved through discussion. Interviewer and analytic biases were managed during regular analysis meetings among all authors. Two study authors (DMM, SB) engaged in regular discussion of cases throughout the data analysis phase to ensure rigor. Transcribed interviews were coded by marked text with phrases indicating content of the discussions. Data appeared to become redundant (i.e., thematic saturation) following the 23rd interview during the pre-adaptive phase and following the 11 th interview during the post-adaptive phase. All authors agreed that no unique responses were emerging within the data and that saturation had been reached. As practitioners had already agreed to participate, seven more interviews were completed during the pre-adaptation phase and three more during the post-adaptation phase.

\section{Maintaining Research Quality}

The Consolidated Criteria for Reporting Qualitative Research (COREQ) framework was used to guide the reporting of findings. ${ }^{11}$ Additionally, criteria for credibility, transferability, and confirmability were used to ensure rigor of this study. ${ }^{12,13}$ Strategies used to address credibility included recording interviews and transcribing them; authors frequently discussing findings; encouraging participants to pursue their own line of thinking; and searching the data for conflicting patterns. ${ }^{12,14}$ Confirmability was addressed by rigorous review of interview transcripts, the codes used to identify them, and drafts and revisions of the findings. ${ }^{14}$ 


\section{Results}

Practitioners were primarily female $(91 \%)$, from academic medical centers $(82 \%)$ or community practices $(18 \%)$ in the U.S. The majority of practitioners interviewed were psychologists $(57 \%)$ or social workers $(32 \%)$.

Table 2 presents practitioner demographics.

Table 2. Characteristics of psychosocial providers.

Bright IDEAS Training

Initial Curriculum ( $\mathrm{n}=30$ ) Adapted Curriculum $(\mathrm{n}=14)$

Training cohorts

$2015-2017(n=30) \quad 2017-2018(n=14)$

Gender, n (\%)

Male

Female

Profession, n (\%)

Psychologist

Social Worker

Nurse Practitioner

Therapist

Case Manager

Health Service Location, n (\%)

Academic Institution

Community-based practice
4

26

16

10

1

2

1

24

6
0

14

9

4

0

1

0

12

2

What follows are reports of key themes present in the data organized by three dimensions of the RE-AIM framework identified for qualitative analysis: adoption, implementation, and maintenance. The themes from participants receiving the initial training curriculum are compared with themes from participants receiving the adapted curriculum are compared in Table 4 and discussed below with representative quotes. 
Table 4

Representative quote comparison between pre and post training adaptations.

\begin{tabular}{|c|c|c|}
\hline $\begin{array}{l}\text { RE-AIM } \\
\text { dimension }\end{array}$ & $\begin{array}{l}\text { Study } \\
\text { Themes }\end{array}$ & Representative Quotes \\
\hline & & Initial Curriculum (n= 33) \\
\hline & & (modeled from clinical research protocol) \\
\hline & & $\begin{array}{l}\text { I pick parents that I feel are highly anxious...they're searching for } \\
\text { some type of sense of control. I feel like using the form and } \\
\text { guiding them through it [Bright IDEAS] gives them that. }\end{array}$ \\
\hline & & $\begin{array}{l}\text { "I've been able to implement Bright IDEAS in all different settings. } \\
\text { So, I've done it inpatient, I've done it on the outpatient side and } \\
\text { certainly done it in clinic as well. It's possible. }\end{array}$ \\
\hline
\end{tabular}

\section{Bright IDEAS Initial Training (2015-2017 cohorts, $n=30)$}

Generally, practitioners perceived Bright IDEAS as highly protocolized and required additional "time" to complete all steps of the intervention. Sometimes, practitioners encountered resistance from overwhelmed patients and overlooked the broad applicability of the intervention with clients.

\section{Adoption}

Overall, practitioners found the Bright IDEAS materials to be useful with their clients and helped to organize their clinical work. For example, a social worker stated, "I usually keep the worksheets and I have them take a picture with their phone of the action plan." Similar to the pre-adaptation group, a profile of the ideal patient was identified by one psychologist as:

patients that have a lot of stressors that tends to be ongoing. So, that could either be a diagnosis and they've just started treatment, or that could be longstanding, strained relationships with their family or their partner. In addition to that, I would say families that don't have a lot of social support specifically, family or social support are good candidates for Bright IDEAS.

Practitioners also described introducing Bright IDEAS to families as, "...if there's some anxiety or stress, or the parent is critical about something, I...put them on my sort of mental list of okay, this might be a good idea for Bright IDEAS."

Additionally, practitioners reported increased attempts to use Bright IDEAS, noted by, "since going to the training I think whenever a family approaches me with a need, I consider using the model [Bright IDEAS]."

\section{Implementation}


Overall, Bright IDEAS was used across diverse clinical settings. Specifically, a psychologist reported, "I've been able to implement Bright IDEAS in all different settings. So, I've done it inpatient, I've done it on the outpatient side and, and certainly done it in clinic as well. It's possible." The implementation of Bright IDEAS was reported as being delivered "in multiple settings with multiple patients." Similarly, a social worker recounted, "I work for a nonprofit and we actually are a community-based organization. So, we go into the patient's house...and meet with them in their environment to discuss their problems...[using] Bright IDEAS." Further, practitioners planned time with clients for Bright IDEAS, "one other patient that I actually scheduled just specifically to do Bright IDEAS..."

\section{Maintenance}

Largely, practitioners discussed ways in which they plan to continue to use Bright IDEAS long term. For example, one social worker shared, "I just consider it [Bright IDEAS] to be another very useful tool in my toolbox to use. I plan to just keep using it for families that clearly will benefit from it." The sentiment was also expressed as, "I feel like it [Bright IDEAS] is something I'm going to always continue to use. If I notice that there are certain participants or patients I have that would really benefit from having the goals." Additionally, there were examples of planned internal training, "So, on my team there's two other people, a social worker and a counselor. The plan is to teach them Bright IDEAS."

\section{Discussion}

To the authors' knowledge this is the first systematic qualitative evaluation of the implementation of an EBCCP-recognized intervention. This manuscript describes the application of RE-AIM to identify the adoption, implementation and maintenance of Bright IDEAS in real-world settings. Based on feedback from trainees, modifications were made to foster a more pragmatic approach to intervention delivery. The new training model fostered expanded use and acceptance of Bright IDEAS at the individual level. Modifications were effective in improving practitioner intention and trial of the intervention. Further, practitioners identified opportunities to train colleagues.

There is a clear distinction between the two training groups, indicating a positive response to the adaptations. We believe the difference between the two groups could be partially explained by the fact that behavioral interventions are more difficult to define and standardize because of the inherent interactivity with client characteristics, preferences, and behaviors. ${ }^{15}$ In this case, Bright IDEAS has proven to be efficacious for greater than 20 years yet factors which may affect real world application had not been previously investigated. Adaptions specifically addressed environmental characteristics and behaviors. For example, we advised that Bright IDEAS NOT be formally introduced to families with newly diagnosed children until at least 4 weeks later,

precisely because of a "not now" response upon initial implementation. 5, 6, 8, 10 Lau and colleagues, ${ }^{16}$ observed similar results when examining adolescents and young adults' perspectives on facilitators and barriers to utilization of psychosocial program and found that "starting something new" was a barrier to utilization.

This study revealed moderating factors that may affect adoption more broadly. This finding is not surprising as Greenhalgh et al noted that standard attributes of the intervention will not ensure adoption alone. Rather, the interaction among the intervention, intended adopters, and a particular context that determines adoption 
rate ${ }^{17}$ In this study, Bright IDEAS training adaptations considered practitioner experience and local context. While there was improved adoption, there is still opportunity for progress. Chambers and Norton describe the needed fit between interventions and their settings and suggest ongoing learning about optimal intervention delivery over time ${ }^{18}$ Gathering feedback across diverse clinical settings and contexts should be a continual process to identify factors which affect adoption and implementation.

Bright IDEAS was accepted into the National Cancer Institute sponsored Speeding Research-tested Interventions (SPRINT) program, which is designed to foster, grow, and nurture an innovation ecosystem for interventionists. ${ }^{19}$ Strategies provided by SPRINT experts were designed to assist in positioning Bright IDEAS as a more accessible, available, and user-friendly tool. Obviously, widespread uptake did not occur however future research should design for dissemination early in the planning process, with consideration for the resources and limitations of the patient, practitioner, and system in mind. ${ }^{20}$

\section{Summary}

Taken together, findings reveal promising results that support the ability to improve the adoption, implementation and maintenance of Bright IDEAS thereby increasing national uptake in clinical settings. At the practitioner level, varying perspectives were expressed regarding capacity to incorporate Bright IDEAS into clinical workflow. Though some practitioners found some difficulties early after training with implementing Bright IDEAS in the clinical setting, training adaptions seemed to resolve some of the issues. Specifically, the identification of a patient profile, increased use across diverse clinical settings, and intentions to train colleagues, is encouraging that improvements were experienced up to 12-month post training after adaptation were made to the training.

Intervention maintenance requires continual feedback from practitioners and adaptations which consider the changing clinical setting and needs of patients. Generalizable lessons learned underscore the importance of continual stakeholder engagement and buy-in for implementation assistance to ensure long term maintenance. Longitudinal follow-up post training is imperative to ensuring the sustainability of an intervention; otherwise limited maintenance should be anticipated.

\section{Limitations And Future Directions}

The limitations of this study should be addressed in future research. First, lessons learned from the national organizations devoted exclusively to childhood and adolescent cancer research and practice, may be difficult to generalize to other healthcare domains. Another limitation is the possibility of social desirability bias. That is, some providers may have responded to questions in a manner they thought consistent with the research aims. Future research with other stakeholders, such as institutional leadership and members of patient treatment teams, also would be valuable to understanding factors that affect the dissemination and implementation process.

\section{Conclusion}

This study highlights some of the issues psychosocial providers face when disseminating a new intervention and the steps that can be taken to improve implementation. Further, the attention to the fit between 
characteristics of an intervention and the clinical setting, available resources and knowledge of potential implementers is critical for informing the implementation process about facilitators and barriers and "work arounds" to barriers. For psychologists and social workers, interventions that require shifts in schedule, additional resources, and new knowledge, we found that a blend of implementation strategies that helps to increase compatibility with existing organizational structures is critical for their implementation.

Future pediatric oncology-based psychosocial interventions should build on the current focus of addressing adoption, implementation, and maintenance issues when trials are first initiated and explore the possibilities of interventions that aim to continually adapt to meeting the dynamic nature of the clinical environment. Maintenance requires integration of research-tested protocols into routine clinical workflow and tailored strategies to support dynamic clinical settings across institutions. Longitudinal follow-up post training is imperative to ensuring the maintenance of an intervention; otherwise limited maintenance should be anticipated.

\section{Abbreviations}

APHON: Association of Pediatric Hematology/Oncology Nurses

APOSW: Association of Pediatric Oncology Social Workers

COG: Children's Oncology Group

COMIRB: Colorado Combined Institutional Review Board (COMIRB)

COREQ: Consolidated Criteria for Reporting Qualitative Research

EBCCP: Evidence-Based Cancer Control Programs

NCl: National Cancer Institute

RTIPs: Research-Tested Intervention Programs

SPP: Society of Pediatric Psychology

\section{Declarations}

\section{Ethical Approval and Consent to participate}

The project was approved by the Colorado Combined Institutional Review Board (COMIRB). This study was conducted in accordance with all applicable government regulations and University of Colorado research policies and procedures. Participants provided verbal consent for interviews through IRB-approved protocols.

\section{Availability of data and materials}

The datasets used and/or analyzed during the current study are available from the corresponding author on reasonable request. 


\section{Consent for publication}

Not applicable

\section{Competing interests}

The authors declare that they have no competing interests.

\section{Funding}

Funded was provided by National Institutes of Health (NIH Grant R01 CA 159013) and the National Cancer Institute (R25 CA65520).

\section{Authors' contributions}

OJS, RBN, and EHM conceived and designed the experiment; DMM, MEV, and DLF performed the experiments; DMM, SB, and DLF analyzed the data; OJS, RBN, MEV, and DLF contributed materials/analysis tools; DMM and EHM wrote the paper; OJS and RBN obtained the funding. All study authors read, edited and approved the final manuscript.

\section{Corresponding Author}

Correspondence to Demetria M. McNeal

\section{Acknowledgements}

Acknowledgement is extended to the National Institutes of Health and the National Cancer Institute for funding NIH Grant R01 CA 159013 and R25 CA65520. Megan Morris and Juliana Barnard of the Adult and Child Consortium for Health Outcomes Research and Delivery Science (ACCORDS), University of Colorado Anschutz Medical Campus are acknowledged for their qualitative methodological expertise and review. Allison Kempe, Director of the Adult and Child Consortium for Health Outcomes Research and Delivery Science (ACCORDS), University of Colorado Anschutz Medical Campus is acknowledged for overall project support.

\section{Authors' information}

\section{Affiliations}

\section{Demetria M. McNeal, PhD, MBA}

University of Colorado Anschutz Medical Campus

Division of General Internal Medicine

12631 E. 17th Avenue | Mailstop L15-8405 | Aurora, CO 80045

demetria.mcneal@cuanschutz.edu

\section{O.J. Sahler, MD}


University of Rochester School of Medicine \& Dentistry

601 Elmwood Avenue, Rochester, New York 14642-8777

OJ_Sahler@urmc.rochester.edu

\section{Robert B. Noll, PhD}

University of Pittsburgh

School of Medicine, Department of Pediatrics

Pittsburgh, PA, 15213

rbn1@pitt.edu

\section{Diane L. Fairclough, DrPH}

University of Colorado Anschutz Medical Campus

Department of Biostatistics and Informatics

Colorado School of Public Health

13001 E 17th PI, Aurora, CO 80045 .

Diane.Fairclough@CUanschutz.edu

\section{Megan E. Voll, MS, LPC}

University of Pittsburgh

School of Medicine, Department of Pediatrics

Pittsburgh, PA, 15213

meganvoll@gmail.com

\section{Shubha Bhat, PharmD, MS}

University of Colorado Anschutz Medical Campus

Skaggs School of Pharmacy and Pharmaceutical Sciences

Aurora, CO 80045

SHUBHA.BHAT@CUANSCHUTZ.EDU

Elaine H. Morrato, DrPH, MPH 
Parkinson School of Health Sciences and Public Health

Loyola University Chicago, Maywood, IL 60153

\section{ELAINE.MORRATO@CUANSCHUTZ.EDU}

\section{References}

1. Gordan VV. How to Bridge Research Results to Everyday Clinical Care? Oper Dent. 2017;42(1):1-9.

2. Morris ZS, Wooding S, Grant $\mathrm{J}$. The answer is 17 years, what is the question: understanding time lags in translational research. J R Soc Med. 2011;104(12):510-520.

3. Gyani A, Shafran R, Myles P, et al. The gap between science and practice: how therapists make their clinical decisions. Behav Ther. 2014;45(2):199-211.

4. Sherman EJ, Primack BA. What works to prevent adolescent smoking? A systematic review of the National Cancer Institute's Research-Tested Intervention Programs. The Journal of school health. 2009;79(9):391-399.

5. Sahler OJ, Varni JW, Fairclough DL, et al. Problem-solving skills training for mothers of children with newly diagnosed cancer: a randomized trial. Journal of developmental and behavioral pediatrics : JDBP. 2002;23(2):77-86.

6. Sahler OJ, Fairclough DL, Phipps S, et al. Using problem-solving skills training to reduce negative affectivity in mothers of children with newly diagnosed cancer: report of a multisite randomized trial. J Consult Clin Psychol. 2005;73(2):272-283.

7. Sahler OJZ, Roghmann KJ, Mulhern RK, et al. Sibling Adaptation to Childhood Cancer Collaborative Study: The association of sibling adaptation with maternal well-being, physical health, and resource use. Journal of Developmental and Behavioral Pediatrics. 1997;18(4):233-243.

8. Dolgin MJ, Devine KA, Tzur-Bitan D, et al. Responsivity to Problem-Solving Skills Training in Mothers of Children With Cancer. J Pediatr Psychol. 2020.

9. Glasgow RE, Vogt TM, Boles SM. Evaluating the public health impact of health promotion interventions: the RE-AIM framework. American journal of public health. 1999;89(9):1322-1327.

10. Sahler OJZ, Dolgin MJ, Phipps S, et al. Specificity of Problem-Solving Skills Training in Mothers of Children Newly Diagnosed With Cancer: Results of a Multisite Randomized Clinical Trial. 2013:1329-1335.

11. Tong A, Sainsbury P, Craig J. Consolidated criteria for reporting qualitative research (COREQ): a 32-item checklist for interviews and focus groups. International journal for quality in health care : journal of the International Society for Quality in Health Care. 2007;19(6):349-357.

12. Carnevale FA. Authentic qualitative research and the quest for methodological rigour. Canadian Journal of Nursing Research Archive. 2016;34(2).

13. Chiovitti RF, Piran N. Rigour and grounded theory research. Journal of advanced nursing. 2003;44(4):427435.

14. Ritchie J, Lewis J, Nicholls CM, et al. Qualitative research practice: A guide for social science students and researchers: sage 2013. 
15. Glasgow RE, Lichtenstein E, Marcus AC. Why don't we see more translation of health promotion research to practice? Rethinking the efficacy-to-effectiveness transition. American journal of public health. 2003;93(8):1261-1267.

16. Lau N, Parsa AG, Walsh C, et al. Facilitators and Barriers to Utilization of Psychosocial Care in Adolescents and Young Adults with Advanced Cancer: Integrating Mobile Health Perspectives. J Adolesc Young Adult Oncol. 2020.

17. Greenhalgh T, Robert G, Macfarlane F, et al. Diffusion of innovations in service organizations: systematic review and recommendations. The milbank quarterly. 2004;82(4):581-629.

18. Chambers DA, Norton WE. The adaptome: advancing the science of intervention adaptation. American journal of preventive medicine. 2016;51(4):S124-S131.

19. Gaysynsky A, Vinson CA, Oh A. Development and evaluation of the SPeeding Research-tested INTerventions (SPRINT) training program. Transl Behav Med. 2020;10(6):1406-1415.

20. Chambers DA. Sharpening our focus on designing for dissemination: Lessons from the SPRINT program and potential next steps for the field. Translational behavioral medicine. 2020;10(6):1416-1418.

\section{Figures}

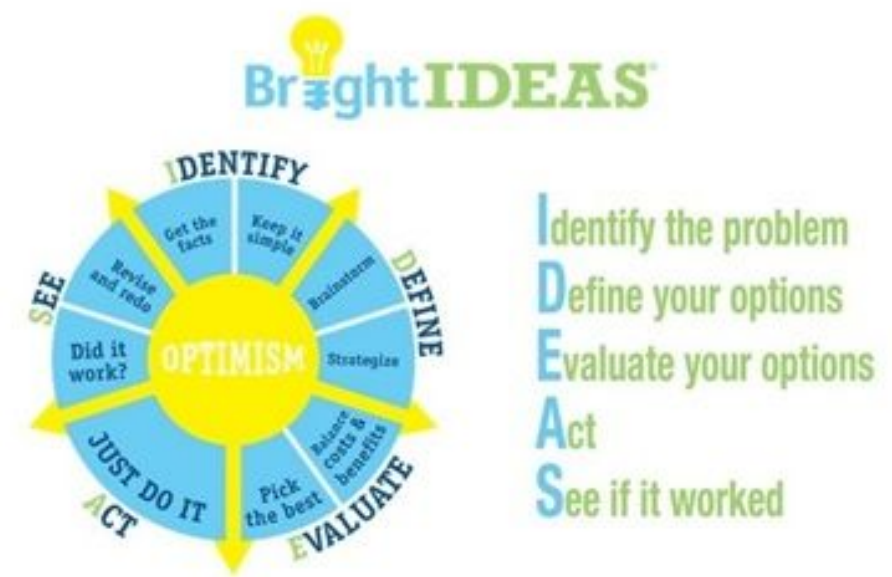

Figure 1

Bright IDEAS Pneumonic.

\section{Supplementary Files}

This is a list of supplementary files associated with this preprint. Click to download.

- AppendixA.WorkshopTiming.preadaptation.pdf

- AppendixB.WorkshopTiming.postadaptation.pdf 
- COREQChecklist5.3.21.pdf

- CoreElementsHandout.pdf 